\title{
The dynamically changing role of CT chest in COVID 19 patients - Plugging the gaps in the guidelines!
}

Sir,

Imaging has made a great impact on the detection, surveillance, and management of the patients in this global pandemic. ${ }^{[1]}$ The editorial was extremely informative and the rationality of using HRCT as a screening tool as against the present recommendations well debated. In continuation with the views put forth in the editorial, we suggest broader indications for $\mathrm{CT}$ as a primary modality in addition to the existing guidelines for COVID-19.
Multiple studies have proven that CT has been used as a standard method for the diagnosis of COVID-19 based on $\mathrm{CT}$ features and rules of transformation. Rapid diagnosis can lead to early control of potential transmission. ${ }^{[2,3]}$

In the scenario of rapidly evolving pandemic the dynamics, the scope, and indications of chest CT as a diagnostic modality is evolving as a tool with high sensitivity due to the low rate $(3.9 \%)$ of missed diagnosis of COVID-19. ${ }^{[2]}$ Chest CT however has a relatively low specificity but can be useful in patients with some 
pre-existing pulmonary diseases. ${ }^{[4]}$ The Coronavirus disease 2019 (COVID-19) Reporting and data system is a categorical assessment scheme for chest $\mathrm{CT}$ in patients suspected of having COVID-19 that simplifies reporting with a five-point scale of suspicion for pulmonary involvement. ${ }^{[5]}$ This has ensured standardization in CT reporting greatly improving its reliability and acceptance among the clinicians.

From a clinical viewpoint, the HRCT chest has been used by the clinicians in quite a few clinical contexts with anecdotal success than what has been prescribed in the guidelines. A set of rationale guidelines based on rational and evidence base has been proposed by various international bodies and radiological societies. Chest imaging has been recommended mainly for patients with moderate to severe features of COVID-19 and evidence of worsening respiratory status. ${ }^{[6]}$ As per the guidelines proposed by the WHO task force, the primary indications for HRCT are for symptomatic patients with suspected COVID-19 when (1) RT-PCR testing is not available; (2) RT-PCR testing is available, but results are delayed; and (3) initial RT-PCR testing is negative, but with high clinical suspicion of COVID-19. ${ }^{[4]}$

We propose three additional indications that have not been proposed earlier with any clarity and specification. These are observations seen as the potential role of HRCT in the broader context of disease with strong indications.

1. Post COVID-19 lung fibrosis as a discrete clinical entity is now increasingly recognized by pulmonologists. Spectrum of pulmonary fibrotic disease observed in COVID-19, ranging from fibrosis associated with organizing pneumonia to severe acute lung injury, with evolution to widespread fibrotic change has been reported with the disease. ${ }^{[7]}$ Even though the recovery in 4 weeks has been seen in the majority of cases, some have shown worsening and few have reported persistence of interstitial fibrosis. Assessment and monitoring the lung fibrosis with baseline HRCT within 2 weeks of the disease followed by interval HRCT between 1 and 3 months will be critical from a medium to long term perspective, more so as spirometry is discouraged in the setting of the pandemic. This is an emerging indication and rapidly gaining ground as we move over to the chronic phase with this pandemic.

2. Development of COVID-19 in patients with underlying interstitial lung diseases (ILD) warrants a strong indication for HRCT upfront for prognostication. Clinical decision making on account of disease overlap, will benefit clinicians hugely as imaging provides information on the likely severity of underlying ILD contributing to the severity of hypoxia.

3. HRCT has been sub-optimally used in most centers in acute emergent situations arising in COVID-19. It is not uncommon to see patients presenting to emergency departments with acute breathlessness and respiratory failure as a primary presentation. Conforming COVID-19 by PCR swab in these situations has issues like technical difficulties in swabbing and practical considerations of reliability of such swabs and time required for the procurement of reports. Both these factors are known to cause delays in the critical emergency decision making process. A screening HRCT incorporated in the protocol can make a difference in the outcome of such cases as a high probability scan (CORADS-5) virtually clinches the diagnosis and overcomes the reliance on microbiological parameters in an acute setting.

These observations are made on anecdotal clinical evidence, case-based practices, and inter-disciplinary experiences shared by pulmonologists, radiologists, intensivists, and microbiologists. Larger scale studies with evidence systematically reviewed, quality of the evidence analyzed for key outcomes, and assessed using the grading of recommendations are mandated to gauge the strength of these proposals.

Financial support and sponsorship

Nil.

Conflicts of interest

There are no conflicts of interest.

Santosh Phajir Vishwanath Rai, Vishak K Acharya ${ }^{1}$, Shreenivasa $A^{1}$, Unnikrishnan $B^{2}$

Department of Radiodiagnosis, ${ }^{1}$ Chest Medicine, ${ }^{2}$ Community Medicine, Kasturba Medical College Mangalore, Manipal Academy of Higher Education, Manipal, Karnataka, India.

\section{References} E-mail: radiorai@gmail.com

1. Kohli A. Can imaging impact the coronavirus pandemic? Indian J Radiol Imaging 2020;30:1-3.

2. Yan Li. Liming Xia. Coronavirus disease 2019 (COVID-19): Role of chest CT in diagnosis and management. AJR Am J Roentgenol 2020;214:1280-6.

3. Czawlytko C, Hossain R, White CS. COVID-19 diagnostic imaging recommendations. Appl Radiol 2020;49:10-5.

4. Use of Chest Imaging in COVID-19: A Rapid Advice Guide. Geneva: World Health Organization; 2020 (WHO/2019-nCoV/ Clinical/Radiology imaging/2020.1).

5. Prokop $M$, van Everdingen $W$, van Rees Vellinga $T$, Quarles van Ufford H, Stöger L, Beenen L, et al. CO-RADS: A categorical CT assessment scheme for patients suspected of having COVID-19-Definition and evaluation. Radiology 2020;296:E97-107.

6. Rubin GD, Ryerson CJ, Haramati LB, Sverzellati N, Kanne JP, Raoof $\mathrm{S}$, et al. The role of chest imaging in patient management during the COVID-19 pandemic: A multinational consensus statement from the Fleischner Society. Chest 2020;158:106-16. 
7. Shi H, Han X, Jiang N, Cao Y, Alwalid O, Gu J, et al. Radiological findings from 81 patients with COVID-19 pneumonia in Wuhan, China: A descriptive study. Lancet Infect Dis 2020;20:425-34.

This is an open access journal, and articles are distributed under the terms of the Creative Commons Attribution-NonCommercial-ShareAlike 4.0 License, which allows others to remix, tweak, and build upon the work non-commercially, as long as appropriate credit is given and the new creations are licensed under the identical terms.

\begin{tabular}{|l|l|}
\hline \multicolumn{2}{|c|}{ Access this article online } \\
\hline Quick Response Code: & \\
\hline & Website: \\
\hline
\end{tabular}

Cite this article as: Rai SP, Acharya VK, Shreenivasa A, Unnikrishnan B. The dynamically changing role of CT chest in COVID 19 patients - Plugging the gaps in the guidelines!. Indian J Radiol Imaging 2021;31:S218-20.

Received: 22-Jul-2020

Revised: 08-Sep-2020

Accepted: 08-Sep-2020

Published: 23-Jan-2021

๑ 2021 Indian Journal of Radiology and Imaging | Published by Wolters Kluwer - Medknow 